\title{
Transferencia de tecnología en regadíos tradicionales y competencia por el agua. México
}

\author{
Acela Montes de Oca Hernández ${ }^{1}$ y Jesús Castillo Nonato ${ }^{2}$ \\ ${ }^{1}$ Centro de Investigación en Ciencias Sociales y Humanidades \\ ${ }^{2}$ Centro de Estudios e Investigación en Desarrollo Sustentable. Universidad Autónoma del Estado de México. \\ acela_cicsyh@yahoo.com.mx,canj19732007@yahoo.com.mx
}

\begin{abstract}
Resumen. El objetivo del artículo es ofrecer un análisis social de la incorporación y utilización de tecnología en sistemas de regadío tradicionales, así como las modificaciones a la cultura de los regantes en México. De manera que, la pregunta es ¿¿de qué manera, la implementación de novedades tecnológicas en regadíos tradicionales provocó y provoca competencias por el recurso? Se responde a dicha pregunta desde la Antropología Histórica, la Política Pública y la propuesta de la acción colectiva. La primera, por la característica temporal del regadío; la segunda, por las líneas de acción del Estado en la administración y distribución del agua; respecto a la tercera, por la respuesta de regantes ante el implemento de tecnología. Mediante Sistemas de Información Geográfica, procesamiento de datos, documentos de archivo oficiales y entrevistas, se llega al análisis de la información. Concluimos en decir que la implementación de tecnología en sistemas de riego tradicionales no tiende a debilitar la acción colectiva, en cambio sí promueve competencia por el recurso lo que acentúa escasez al alterar los mecanismos de distribución de agua, conservación de infraestructura y agropaisajes.
\end{abstract}

Palabras clave: acción colectiva, agropaisaje, cultura, tecnología, riego.

\begin{abstract}
The aim of the article is to provide a social analysis of the incorporation and use of technology in traditional irrigation systems, and changes to the culture of the irrigators in Mexico. So, the question is how the implementation of technological innovations in traditional irrigation, caused and causes competition for water? We answer the question form de proposal Historical Anthropology, Public Policy and the collective action. Regarding the historical anthropology by temporal characteristics of irrigation; the Public Policy, by the lines of action of the State in the administration and distribution of water; regarding the collective action by the response to the implement irrigation technology. Basins using geographical information systems, through review of documents for the archiving, interviews and observation obtained the analysis of information. We conclude in saying that the implementation of technology in traditional irrigation systems do not tend to weaken collective action, however, promoting competition for the resource shortage which stresses by altering the distribution mechanisms of water conservation infrastructure and agroscape.
\end{abstract}

Keywords: collective action, agroscape, culture, technology, irrigation.

\section{Introducción}

La antropología mexicana a mediados del siglo XX empezó a plasmar en sus estudios la importancia del regadío en la cultura de los pueblos mesoamericanos. Desde la arqueología se mostró a través de los estudios de Armillas y Carrasco que la infraestructura hidráulica fue un referente para entender el comportamiento de los sistemas de producción agrícola por las culturas de Mesoamérica. A la par de estos estudios, destacarían las aportaciones de Palerm y Wolf, quienes se interesaron en el tipo de organización sociopolítica emergida a través del uso y manejo del agua, en sus estudios se visualiza el encadenamiento de grupos sociales surgidos en relación con la defensa o control de sistemas hidráulicos. Dichos principios de estudio marcaron un interés por entender el comportamiento de las sociedades campesinas en un determinado territorio, así que Palerm (1972) a través de las 
investigaciones de documentos históricos, recorridos de campo y cartografía del Valle de México descubre la importancia de los sistemas hidráulicos para las sociedades; sus estudios revelaron que en México existía una tradición milenaria de construcción de obras hidráulicas de regadío, sin embargo, lejos de considerar un centralismo del Estado con la construcción de dicha obras, lo que le interesó fue mostrar la eminente organización social emergida del regadío; por lo tanto, la agricultura de riego permitía a los grupos generar excedentes y desarrollar una cultura urbana, además de promover que amplios sectores de población pudiesen, dados los altos rendimientos, desligarse del trabajo agrícola (Palerm, 1972; Palerm y Mestre, 2007).

Por otra parte el regadío trajó consigo la emergencia de poder, evidenciado en la estructura de las autoridades así como en la toma de decisiones respecto a las reglas de apropiación de agua y tierra. En este sentido, en la Constitución de 1917 se establece en el artículo 27 que "La propiedad de las tierras y aguas comprendidas dentro de los límites del territorio nacional, corresponde originariamente a la Nación, la cual ha tenido y tiene el derecho de transmitir el dominio de ellas a los particulares, constituyendo la propiedad privada". En este tiempo más del $90 \%$ de las familias carecían de propiedad de tierra y agua, años más tarde y con severas restricciones jurídicas y políticas se establecen garantías de la propiedad y se establecen derechos de propiedad comunal y ejidal (Derecho Agrario).

Estos avances, en materia jurídica, trajeron consigo la aplicación de innovaciones tecnológicas en el campo agrícola mediante el incremento e inserción de sistemas de regadío. Así que nuevos y viejos regadíos en principio mantuvieron una expectativa sobre los incentivos financieros aplicados en la infraestructura hidráulica para hacer de estos paisajes agrarios sistemas de producción competitivos. Sin embargo, los proyectos hídricos de los organismos del Estado destinaron la construcción de obra hidráulica generalmente en terrenos que no presentaban problemas orográficos, en este sentido el contexto territorial ha favorecido a algunos y desfavorecido a otros. En estos últimos, las condiciones no favorables del suelo, relieve y clima han conducido a que los pobladores potencialicen el uso del suelo mediante el aseguramiento del agua y diversidad de cultivos, actividades preponderantes en espacios agrícolas con regadíos tradicionales; éstos han sido obviados tanto por parte de las instituciones sectoriales como por las territoriales, por lo que se carece, en general, de una normativa para su conservación, ordenación y gestión (Castro y Torregrosa, 2004; Mata, 2004; Silva, 2008). En los paisajes de regadío tradicional existe una conexión directa entre la tierra, agua y actividades productivas, lo que genera un reforzamiento de las organizaciones locales asociado a las prácticas de uso y manejo de agua y tierra.

Aparentemente las organizaciones sociales de regadíos tradicionales se encuentran físicamente desvinculadas, sin embargo, éstas se encuentran enlazadas por la propia dinámica de circulación del agua de ríos, manantiales y/o arroyos. Fundamentalmente estas organizaciones han permanecido por la vinculación con el territorio hidráulico que les provee de seguridad alimentaria además de los mecanismos y reglas organizativas locales que les permiten regular el recurso.

Desde el discurso de algunos actores políticos y sociales, las acequias tradicionales han sido demonizadas como deficientes por las siguientes razones: malgastan el agua, son fuentes de contaminación, no existe una regulación y entrega de agua cuantificable, la disponibilidad del agua depende del caudal del río y no de la demanda de los usuarios, por ejemplo. Pero aquí sucede algo muy curioso, la idea dominante ahora entre los agrónomos es que se debe 
terminar con las acequias superficiales, entubar el agua y hacer riego de goteo. Aquí hay dos problemas. El primero interfiere con la recarga natural de recursos acuíferos, que se hace a través de la filtración de agua en estos campos de riego. El segundo se relaciona con la evapotranspiración. El agua se evapora, permitiendo la lluvia (Glick, 2007).

Algunas investigaciones han demostrado que en estas últimas décadas, en cuanto se transforman las superficies con regadíos funcionales por la expansión de la ciudad o la desecación de tierras marjales, el resultado es menos lluvia. Esto resulta contraproducente. Si se prosigue con la entubación, la lluvia será aún menor y caerá en lugares distintos de aquellos en los que caía anteriormente. Métodos más tradicionales de agricultura, como las acequias usadas en la Vega de Granada, han servido de método de compensación a la naturaleza en sus procesos climáticos, mientras que en la actualidad el vapor de agua acumulado sobre el Mediterráneo no es suficiente para evitar inundaciones en el centro de Europa y el sur, en el invierno, mientras disminuye la cantidad de precipitaciones suaves en otoño (Glick, 2005, Gozzer, 2010).

Lo que sugerimos es una aproximación a los regadíos tradicionales, característicos de regiones con población rural, indígena, mestiza, campesina y pobre; ellos, a partir de 1926, asumieron un papel importante en la política centralizadora del agua; estos espacios fueron confinados para ser administrados por el gobierno federal, a raíz de este proceso, algunos de ellos se han tecnificado; la tecnificación de regadíos tradicionales obedece fundamentalmente a que son concebidos como obsoletos, con bajo rendimiento y pobre calidad de productos agrícolas, además de que no coadyuvan en satisfacer demandas nacionales (Cedillo y Calzada, 2005).

De manera que, la adopción de tecnología, por medio de la tecnificación del riego o la transferencia de recurso, ha sido uno de los esquemas instrumentados para logar posicionar al sector rural en la economía regional, nacional o mundial. Sin embargo, este tipo de proyectos focaliza los apoyos en productores rurales con capacidad económica, de manera que su promoción alienta la competitividad interna por tierra y agua, la tierra por la expansión de cultivos de renta y el agua por la requisición puntual del recurso de productos agrícolas comerciales y/o el desvío de agua en fechas requeridas por el propio cultivo.

En México, en la segunda década del siglo XX, la política hídrica se enfocó en aumentar la superficie de riego e incrementar la producción agrícola con la inversión federal en obra de gran envergadura, por ejemplo, las presas; dichas obras han servido, entre otros usos, para la construcción de obras de irrigación, generando sistemas de regadío altamente tecnificados, ubicados en mayor proporción en el norte del país. Por su parte, los estados con marginación social y productiva, ubicados en la parte sur y centro de México, se caracterizan por la continuidad del regadío tradicional, aunque también presentes, en menor medida, en el norte del país. De manera que, dicha polarización de sociedades requiere analizar los mecanismos culturales en regadíos tradicionales ante las innovaciones técnicas de riego.

Así, este artículo se divide en tres apartados: el primero se refiere a la configuración del territorio mexicano y la importancia del regadío tradicional; el segundo apartado relaciona los siete principios de organización comunitaria en regadíos tradicionales; mientras el tercero aborda los implementos tecnológicos en casos de Distritos de Riego y un Distrito de Desarrollo Rural, por último, se estima la transferencia de tecnología en la competencia por el recurso tierra y agua. 


\section{Metodología}

Se seleccionaron casos de estudio de distritos de riego y un distrito de desarrollo rural en la parte centro sur del Estado de México, por el avance desmesurado del incremento poblacional, el crecimiento de la industria y la presencia de regadíos tradicionales. Este trabajo parte de una investigación histórica, de manera que emplea fuentes documentales extraídas de archivos nacionales y estatales dedicados al tema del agua. Además se realizó trabajo de cartografía para comparar las extensiones de espacio dedicado al regadío frente a los sistemas agrícolas de temporal. Se realizó consulta de datos de fuentes de organismos oficiales para la obtención de datos respeto a transferencia de tecnología en sistemas de regadío.

\section{Importancia política, social e hídrica en México}

La parte sur México se sitúa en la zona intertropical de la tierra, mientras la norte se localiza en la zona templada el eje del meridiano, lo que le permite contar con climas y microclimas variados que favorecen la diversidad biológica de cultivos y de sistemas productivos. Dos terceras partes del territorio se consideran áridas o semiáridas con precipitaciones anuales de $500 \mathrm{~mm}$, en tanto que el sureste es húmedo con precipitaciones promedio que superan los 2,000 $\mathrm{mm}$ por año. Históricamente este país se ha integrado por diversos tipos de población que habita en 2,440 municipios y 16 delegaciones, a su vez en ella se encuentran 187,938 localidades con un desigual repartimiento de tierra y agua. Más del $50 \%$ de su población está catalogada como pobre, dentro de este estudio se dividirá este sector en pobres con tierra y pobres extremos con o sin tierra y sin riego. En este contexto, cobra especial relevancia el hecho de que México ocupa el sexto lugar mundial en términos de superficie con infraestructura de riego con 6.46 millones de hectáreas repartidas en un 58\% y $42 \%$ de grandes y pequeños sistemas de riego. En este espacio geográfico se encuentran distribuidas las obras de riego nacionales que compiten con otros usos del agua como la doméstica y la industrial, por ello a estos datos ingresamos la cantidad de plantas tratadoras de agua para comparar la prominencia de la política hídrica en el tema del procesamiento del agua residual.

Tabla 1. Infraestructura Hidráulica en México. 2010

\begin{tabular}{lcc}
\hline Obra & Cantidad & has \\
\hline Presas & 3,377 \\
$13,000-10,000 \mathrm{hm}^{3}$ & 3 & \\
$10,000-5,000$ & 2 & \\
$5,000-1,000$ & 19 & \\
$1,000-\quad 500$ & 11 & \\
$500-\quad 100$ & 39 & 2.74 \\
$100-\quad 40$ & 3,276 \\
Menos de 40 & 1,085 \\
Bordos ${ }^{1}$ & 604 \\
Temporal tecnificado & \\
Planta de tratamiento de aguas & 2,082 \\
residuales (municipales) & \\
Plantas de tratamiento de residuales & \\
(industriales) & \\
\hline Fuente: Comisión Nacional del Agua (2010) e INEGI (2010).
\end{tabular}

\footnotetext{
${ }^{1}$ Se cuenta con un registro incompleto de bordos.
} 
En la parte noroeste y pacífico norte de ubican las presas con mayores volúmenes de almacenamiento representando el $20 \%$, en la parte noroeste y golfo norte se localizan las de dimensión mediana y pequeña, estás últimas en términos porcentuales responden al $80 \%$. Las presas medianas y pequeñas han recibido menor recurso financiero para mejorar la eficiencia en el aprovechamiento del agua, lo que ha representado junto con el aumento de los costos de producción un abandono paulatino del campo agrícola y la migración de la fuerza de trabajo masculina en las últimas décadas. Continuando con ese reconocimiento del espacio de riego tradicional, las localidades de los municipios de la República Mexicana que están insertas en este grado de rezago social, de manera aleatoria se han establecido en entornos ambientales donde el uso del recurso hídrico ha generado conflicto por el cambio de uso de suelo, lo que ha influido en sus condiciones de vida. Sin embargo, el agua y la tierra han sufrido constantes transformaciones, algunas veces promoviendo el desarrollo económico y en otros casos disminuyéndolo o alterándolo.

Los espacios de riego tradicional han dado origen a diversos segmentos territoriales en localidades rurales, las extensiones de tierra definidas por el relieve y por la cantidad de agua almacenada en las presas o la presencia de los ríos originan un paisaje heterogéneo, por una parte, la agricultura bajo riego tiene relación con la existencia de bosques mixtos y, por otra, la presencia de espacios de regadío tradicionales ubicados en la parte centro sur de la República Mexicana. En el caso del Eje Neovolcánico, su topografía impide el aumento de extensión del riego, existiendo una porción de paisaje agrario con riego eventual. Predominan de esta manera las tierras de temporal por sobre las de riego, sin embargo las tierras de temporal se ubican en las zonas de laderas y montaña, por su parte las tierras de riego ocupan los valles y pendientes ligeras. El mapa siguiente muestra en un espacio situado segmentación de los espacios de regadío a razón de las condiciones físicogeográficas.

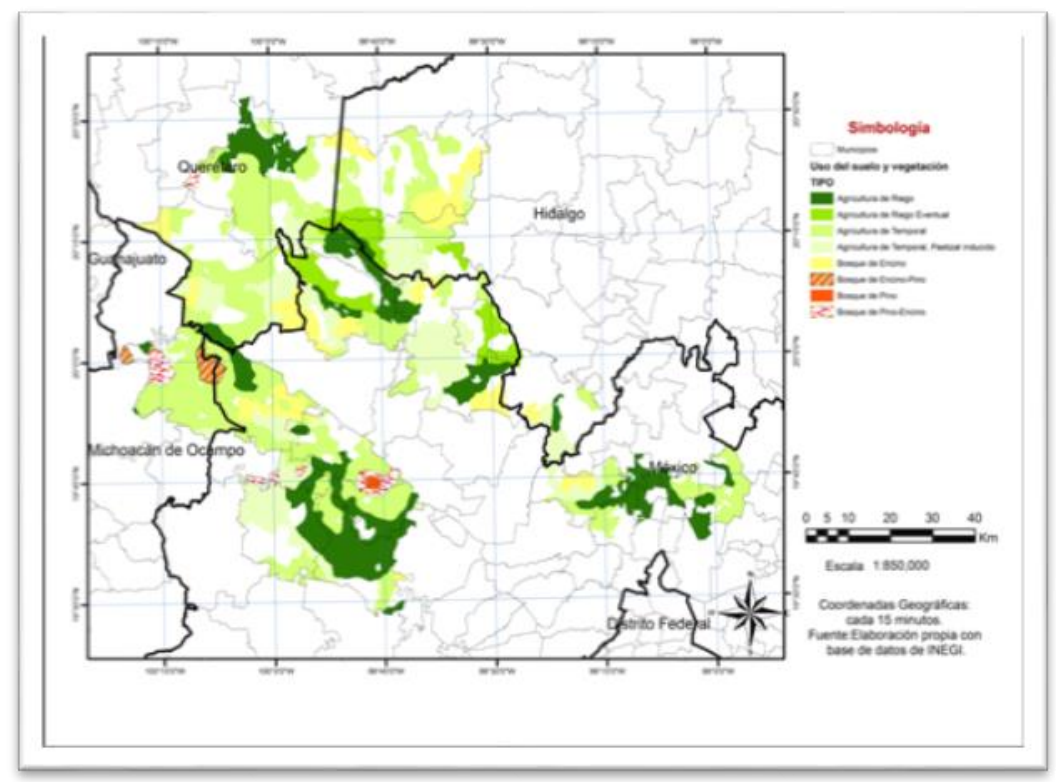

Mapa 1. Zona de riego y temporal en espacios rurales del Estado de México. 2014.

En el mapa se representan la totalidad que ocupan los espacios de riego tradicional en uno de los Estados de la República Méxicana, éstos fueron administrados por el personal del 
Estado por medio siglo, recientemente (2000) fueron entregados a organizaciones de usuarios conservando la categoría política de Distritos de Riego (DR). Los DR, sin perder su ámbito de administración territorial (presa), a partir del año 1988, quedan circunscritos a los Distritos de Desarrollo Rural (DDR). Los DDR surgen como base territorial para la implementación de los programas operativos de los sectores de producción rural. En ambas unidades de gestión territorial se estima el valor de la producción agrícola vinculados a la provisión de agua para riego.

Los DR se crearon en la segunda década del siglo XX, el objetivo del gobierno fue impulsar la producción de alimentos e iniciar el comercio agrícola internacional, así que lo consideramos como el primer proyecto hidráulico federal en materia nacional. Con la creación de los DR, la mayoría de los estados de la República fueron incluidos en el proyecto nacional, en ellos se implementó un plan hídrico cuya única autoridad representante en la administración del sistema hidráulico fueron empleados federales de la Comisión Nacional de Irrigación, posteriormente la Secretaría de Recursos Hidráulicos, mas tarde la Secretaría de Agricultura y Recursos Hidráulicos y, a partir de 1992, la Comisión Nacional del Agua en mancomunidad con Asociaciones de Usuarios y/u otros organismos operadores.

Los DR no coinciden geográficamente con la delimitación de una cuenca hidrográfica tampoco con límites municipales, es una regionalización basada en cuerpos de agua, en cuyo interior funcionan varios sistemas de riego de menores dimensiones diferenciadas por fuentes de suministro de agua $(\mathrm{FAO}, 2000)$.

Los DR adoptaron una política de agregación territorial de varios almacenamientos, lo que condujo a la incorporación de campesinos con realidades económicas, políticas y productivas diversas. Los DR cubren una superficie de riego de 300,000 a 1,000 has, éstos a su vez se dividen en módulos de riego cuyas extensiones varían entre 800 y 1,000 has. Las localidades o municipios beneficiados con las obras son sólo aquellos circunscritos al área de almacenamiento y cuyo volumen de almacenamiento de la presa logre abastecer.

Por su parte las zonas que integraron un DDR comprendieron ciertas características homogéneas en términos físicos y productivos, de manera que se buscó incrementar la producción en todos los municipios de los diversos estados de la República. En el caso del Estado de México figuran seis DDR, que se integran con la totalidad de los municipios. Bajo estos proyectos hídrico-productivos nacionales se buscó compatibilizar los objetivos de los DDR con los DR, fungiendo los primeros como coordinadores de proyectos. Entonces, los DDR surgen como unidades de gestión para el desarrollo, siendo el centro que implementa los programas operativos de transferencia de tecnología, así como los conveníos de apoyo entre las tres órdenes de gobierno. Uno de los problemas más graves en los DR, con mayor prominencia en los DDR, es el uso de aguas residuales para riego.

\section{Sistemas tradicionales de riego}

En lo que respecta a la superficie bajo riego en México, hasta el 2000, el 92.3\% utilizaba riego tradicional y únicamente el 7.7\% tenía algún tipo de riego tecnificado (CNA, 2002). El riego tradicional consiste en llevar el agua por gravedad dentro de la parcela, desde la visión de los ingenieros éste se caracteriza por su baja eficiencia ( $40 \%$ en promedio, que incluye la conducción, la distribución y el riego parcelario), utilizado principalmente en 
cultivos básicos mejorados como maíz, trigo y arroz, así como en caña de azúcar y en algunas hortalizas. El riego tecnificado incluye al riego por gravedad mejorado (sifones y multicompuertas), riego por aspersión y al riego localizado (goteo), se caracteriza por una alta eficiencia de aplicación de agua (más del 90\%), promueve salinidad de suelos, requiere de insumos energéticos que elevan el costo de producción, generalmente recomendado para cultivos de alto valor comercial como las hortalizas, flores y algunos frutales.

En México, los sistemas de regadío tradicionales han sido relevantes por contribuir a disminuir a escasez de alimentos en poblaciones rurales y marginadas, así como también en proveer de alimentos mayoritariamente orgánicos a la región donde éstos se encuentran. Pero ¿qué es un sistema tradicional de riego? En términos generales diremos que el regadío tradicional en México se caracteriza por algunos de los siguientes elementos: presencia de administración autogestiva, existencia de un reglamento interno y continuidad en la aplicación del mismo, figuras administrativas tradicionales, medidas de agua prehispánicas y/o coloniales, marcas de medidas derivadas de las mediciones del cuerpo humano (brazos, pies) para organizar y distribuir entre los usuarios, labores de limpieza comunitaria de un canal de riego, sistemas de cultivo mesoamericano y colonial (milpa, solar, huerto), antecedente de trabajo mancomunado de beneficio comunal y/o agua rodada, principalmente superficial. Para Glick (2013), los regadíos tradicionales mantienen el mínimo de conflicto por el agua gracias al sistema político de autoridades consensuadas.

Desde la parte operativa, dichos sistemas han tenido como particularidad mayores horas de trabajo manual para irrigar un campo de cultivo, de manera que, el jornal laboral de riego consiste en "ir a traer el agua" desde un punto establecido, usualmente una compuerta, hasta el terreno mismo. Dicha labor implica dosificar y controlar el paso del agua por los canales para evitar las inundaciones a otros terrenos, así como evitar la alteración en el tandeo de distribución.

La eficiencia en la distribución del agua en estos sistemas depende en menor medida de la tecnológica y en mayor medida de la organización social; el cumplimiento de reglas, la flexibilidad en los tiempos y la gestión oportuna del recurso ante la federación, es lo que evita la insuficiencia en el recurso. Otro de los elementos interrelacionados congruentemente con el agua ha sido la tierra. Este recurso ha sido parte eminente en la composición estructural de los espacios de riego.

La política hídrica del siglo XX originó dos tipos de repartimiento: el primero de tierras, y el segundo de tierras y aguas. El reparto agrario no intensificó los sistemas de riego tradicionales, por el contrario promovió numerosos usuarios para un mismo aprovechamiento, así que existió una extensión de propietarios usufructuarios el agua. La aparición de nuevos usuarios conllevó a la creación de nuevos reglamentos de agua y nuevos modelos de organización social. Diversas localidades fueron integradas en un solo sistema de riego, dando origen jurídico a organizaciones de usuarios denominadas Juntas de Agua y Asociaciones de Regantes. El principal objetivo de dichas organizaciones era el aprovechamiento colectivo, de manera que aquellos grupos quedaban ligados entre sí no sólo por un aprovechamiento común, sino por la forma en que le fue otorgada la tierra.

En función del lugar y la cantidad de terreno que tenían los usuarios era determinado el tiempo que gozarían de riego. En principio, los nuevos usuarios requirieron de experiencia para aprender a distribuir el agua, por su parte, los viejos usuarios trataban de que sus anteriores repartimientos no fueran alterados. El problema que se planteó en el siglo XX 
fue igualdad en la distribución de agua en aquellos espacios donde existían principios de organización y aprovechamiento, así como las nuevas aplicaciones de reglamento establecidas por el gobierno federal. Dichas realidades sociales en el riego se han sustentado en organizaciones autogestoras que evidencian principios de acción colectiva, con presencia en DR.

\subsection{Límites claramente definidos}

En los sistemas de regadío tradicionales prevalecen ciertas características definitorias relacionadas con la inclusión y exclusión de las comunidades de regantes de alguna de las tareas que requiere el propio sistema de regadío. De manera que, el derecho que se demanda respecto a las fuentes de agua, su infraestructura hidráulica, así como rehabilitación y/o acondicionamiento, tiene que ver con el número de extensión de has que se riegan y con el origen de la fuente de agua. Se trata de encontrar entre los diversos usuarios una proporcionalidad entre la cantidad de agua recibida y la aportación de trabajo sobre la red hidráulica. En este caso los límites están establecidos por tres factores: los límites políticos del poblado, el lugar donde se ubican los terrenos de riego y la infraestructura de riego que provee de líquido al cultivo.

Cada una de las localidades debe atender al tema de cuidado de la infraestructura hidráulica en todo el sistema, la incorporación de trabajo colectivo, sin embargo, no siempre sigue este principio rector de definir claramente sus límites, es decir, la organización autogestiva en regadíos tradicionales tiene flexibilidad en atender lo que se considera su espacio de riego, de esta manera se fortalecen en algunos casos los lazos comunitarios y en otros se promueve competencia entre comunidades por el aprovechamiento de agua y manipulación de las compuertas.

\subsection{Coherencia entre las reglas de apropiación y de provisión}

Las reglas de apropiación refieren a los usuarios incluidos o no en el padrón de riegos para tener acceso al agua; es importante que los usuarios se registren y emitan datos a sus autoridades del tipo de cultivos y número de has irrigadas durante los ciclos de riego ( $\mathrm{P}-\mathrm{V}$ y $\mathrm{O}-\mathrm{I})$, ya que los datos registrados permiten generar un calendario de riegos en función de la cantidad de agua que la gerencia de DR autorice y la demanda de todos los usuarios.

La representatividad de cada una de las localidades de riego o de pequeños propietarios ante la organización de usuarios permite que se asegure la cantidad de agua requerida, así como el establecimiento del lugar, día y hora en que se entregará el volumen de agua correspondiente.

\subsection{Arreglos de elección colectiva}

A pesar de que algunos DR en sus respectivos módulos o único módulo aceptaron la transferencia como lo marcaba la legislación, durante su mandato flexibilizan lo dispuesto por la normatividad y lo marcado en el título de concesión. Es decir, modifican las acciones que tiendan a afectar a la mayoría de los usuarios, tal es el caso de la fijación de cuota de riego; ésta se establece en función del promedio de has y tipo de cultivo que la mayoría de 
usuarios posee, así que dependiendo la zona agrícola y el destino de producción, las cuotas fluctúan de $\$ 450.00$ a $\$ 10.00$. Temas relacionados con la limpia de canales de riego, rehabilitación de infraestructura hidráulica, construcción de nueva obra (puente-canal), elección de autoridades y multas por infringir los acuerdos se discuten en asambleas comunitarias, de manera que los acuerdos son sancionados y aprobados por la colectividad.

\subsection{Sanciones graduadas}

Otra forma de castigar a los infractores desde el siglo XX ha sido la disminución en el volumen de agua. Cuando los usuarios se negaban a aportar la cantidad de dinero fijada por las autoridades (juntas de aguas) para la reconstrucción de presa o canales, éstas establecían sanciones como privación de dos horas de riego a los usuarios morosos, reactivándoles la totalidad de horas a que tenían derecho una vez cubierto su pago. Esta acción, sin embargo, no era aceptada por la Dirección general de Aprovechamientos Hidráulicos de la SAy F (AHA, AS, caja 2410, expediente 34136, Foja 40).

Ahora, difícilmente las organizaciones sociales con regadíos tradicionales establecen como correctivo de primer orden un castigo que dañe la libertad y/o economía de los usuarios, basta con un reconocimiento público del infractor; dicha acción está basada en acuerdos comunitarios que establecen que la primera sanción debe ser verbal, si éstos persisten, entonces se lleva el caso a la asamblea y se juzgan de acuerdo al reglamento interno (establece sanciones económicas y reparo del daño), en caso de reincidir, los representantes de los usuarios lo/los remiten a la delegación municipal o bien, en casos de continua reincidencia, las autoridades emiten una demanda en la presidencia municipal.

\subsection{Mecanismos de resolución de conflictos}

Los conflictos por el acceso y conservación del agua para riego presentan variaciones, primero porque al iniciar el año y con él el ciclo de riego, las autoridades deben atender el tema de robo de agua, es decir, atender a aquellas localidades o usuarios que determinen regar en tiempos que no les corresponden, en este caso existen resolución de conflictos al interior de la localidad y al exterior de las mismas. Antes de iniciar el riego deben de monitorear que los canales estén limpios para evitar que la basura impida el libre flujo del agua, además de cooperaciones que tienen que ver con la celebración de misa (en localidades con presencia de población originaria) en el lugar donde emana el agua para riego. Aquellos usuarios que no realizan su labor de limpia de canales se les impone una multa económica, además de que socialmente queda restringida su participación en asambleas en la toma de decisiones; a veces las reglas son flexibles y les es permitida su asistencia, pero carece moralmente de voz, de manera que automáticamente queda restringida su participación para ser electo autoridad del riego. Toda vez que inicia el riego, los conflictos inician entre localidades: usuarios aguas arriba contra usuarios aguas abajo. Los primeros se quejan de aprovechar el remanente de agua que dejan los usuarios con extensiones de cultivos mayores, los segundos mencionan que la tardanza en el cierre de compuertas, toda vez que termina el tiempo de riego, provocan desperdicios de agua. En ambos casos se llega a acuerdos informales porque sólo tienen aplicación en el momento de conflicto y ayudan a disminuir la escalada del mismo, además de que sólo participan los afectados. En estos casos se forman comitivas de usuarios (grupo de 10 a 15 usuarios) que exigen y presionan a los usuarios aguas arriba para que se ajusten a las fechas de riego 
establecidas, los acuerdos, cuando las fechas de riego han sido alteradas son: que usuarios aguas arriba aprovecharan la mitad del volumen de agua y la restante la dejaran para los usuarios aguas abajo, de manera que las horas de riego entre ambos usuarios disminuye. Es decir ambos usuarios son sujetos a restricciones en un primer ciclo de riego, sí en los subsecuentes ciclos continua la falta e incumplimiento a las fechas se recurre a la destitución de las autoridades por su incapacidad para ordenar los riegos.

\subsection{Supervisión}

Los sistemas de riego tradicionales son supervisados por los mismos usuarios, sean o no autoridades de riego, la finalidad es presionar a las comunidades aguas arriba a que no retrasen el paso del agua, por no atender a las fechas establecidas "desde tiempo inmemorial" o "por costumbre", lo que significa que a cada localidad se le asignan días de riego con sus respectivas horas; las localidades están pendientes del tiempo de riego y si éste se retrasa en horas o días van directamente a los puntos de control, si aún las compuertas están abiertas en fechas tardías estas son cerradas (con presencia de un representante del riego), dirigiendo (con autorización del delegado de riego y del comisariado ejidal) el agua a las comunidades afectadas (ejido o pequeña propiedad). Normalmente los usuarios están vigilando a los pequeños propietarios o ejidatarios con mayor número de has de tierra, porque son los que detentan mayores cantidades de agua, por tiempos mayores a los establecidos.

\subsection{Reconocimiento mínimo de derecho a la organización}

Los usuarios en este tipo de regadíos no presentan una organización acéfala, se rigen por la normatividad de la Ley de Aguas Nacionales y su reglamento, donde se establece que la Nación es la única dueña de la propiedad de tierras, agua y bosques, de manera que eligen usualmente a tres representantes de agua, basados en los reglamentos de aguas del siglo XX o bien por costumbres ibéricas. Dichas autoridades se rigen por dos leyes: las que emergen de la propia colectividad y las que exige la federación. Por lo tanto, para tener un orden en el acceso al agua entre diversidad de actores se amparan en sus autoridades y reconocen que su alineación a las reglas establecidas en la propia organización fundamenta la existencia del riego mismo. La organización en el riego es otro elemento de igualdad en el acceso al agua, porque se determina un rol de riegos que varía entre 15, 2030 y 45 días, en función del número de usuarios que integra la organización y es parte del sistema. Toda vez que el riego ha llegado a todos los usuarios registrados nuevamente regresa el orden del riego al punto donde inició, para dar un segundo riego. Dicho control en el riego sólo puede ser detentado por una organización con su estructura perfilada para definir las funciones de los actores. Esquemáticamente reciben agua los usuarios que previamente se han registrado en dicha organización. Usuarios que no reconocen a sus autoridades y no forman parte de una organización, pero que utilizan en algún momento el agua, promueven alteraciones sociales que se incrementan por las presiones sobre el recurso. 


\section{Huella de transferencia de tecnología en sistemas de riego tradicional}

Transferencia de tecnología refiere a la transmisión del saber hacer (Know-bow) de conocimiento (Segura, 1978). En este sentido, en los DR, la incorporación de tecnología se suministró en 1926 a través de la Comisión Nacional de Irrigación, posteriormente la Secretaria de Recursos Hidráulicos, la Secretaria de Agricultura y Recursos Hidráulicos y, a partir de 1992, por la Comisión Nacional del Agua. Para tener derecho a ser partícipes de los apoyos gubernamentales, las Asociaciones de Regantes deben estar reconocidas por derecho jurídico (registradas ante la Secretaría de Relaciones Exteriores) y contar con título de concesión, obligados los usuarios a pagar un derecho por el uso del agua, por la explotación que hacen de la misma.

Al amparo de los títulos de asignación otorgados por la Comisión Nacional del Agua, se plantea el goce de este recurso de dominio público además de ser sujetos de apoyos gubernamentales; La Comisión Nacional del Agua como autoridad fiscal programa mensualmente las recaudaciones por volumen de agua concesionada, de manera que a la organización registrada es a quien corresponde realizar el pago en la Secretaría de Hacienda y Crédito Público. No todos los DR con sistemas de regadío tradicional cuentan con reconocimiento jurídico, lo que limita su participación. De manera que, en un primer momento, no se proyectaron obras hidráulicas en espacios rurales con tradición organizativa, lo que se hizo fue aglutinarlos para construir nuevas organizaciones, en estos espacios, el paisaje agrario se modificó en cuanto a las obras de distribución; éstas se avocaron en abrir nuevos canales de riego para beneficiar a nuevos regantes; al menos en papel, el objetivo fue incrementar la superficie de regadío.

La consideración de que el regadío por sí sólo no cumpliría los objetivos de mejora de la productividad agrícola, promovió que los programas gubernamentales se centraran en el implemento de mejoras en la producción agrícola, así que los campesinos fueron aleccionados en la implementación de paquetes tecnológicos en sus cultivos. No todos los agricultores adoptaron dicho esquema, porque las inversiones en la producción agrícola los degradaban económicamente, por ello, paulatinamente han dejado que el mercado de renta de tierras aminore los problemas del agro mexicano. Aquellos agricultores que han adoptado la asistencia técnica e invertido económicamente en la reconversión de cultivos, constantemente participan en los convocatorias de apoyos federales al campo, incrementan sus extensiones incorporando más de 50 has a la agricultura intensiva.

Sin embargo, dicha voluntad de cambio e incorporación de tecnología agrícola ha visto amenazado el crecimiento productivo y económico de los campesinos por las condiciones de mercado, lo que ha limitado su participación en este proceso de transferencia tecnológica. Por otra, parte los cambios en los patrones de cultivos afectan la distribución del agua entre los mismos propietarios comerciales y los tradicionales. La acaparación de agua por los agricultores comerciales (cisternas, bordos) ha sido uno de impedimentos para que los campesinos tradicionales obtengan el agua en el tiempo y cantidad adecuada, originando fechas tardías de siembra, éstas vulneran la producción agrícola por la presencia de heladas o lluvias tardías, llegando inclusive a perder la cosecha o dejarles inahibilitados durante uno o tres ciclos agrícolas.

En el caso de los DDR, la incorporación de apoyos gubernamentales en el regadío se establece a través de los programas federales a cargo de la Secretaría de Agricultura, Ganadería, Desarrollo Rural, Pesca y Alimentación (Sagarpa), cuya participación de 
transferencia de tecnología se especifica de acuerdo con las reglas de operación del propio programa. Las instituciones encargadas de difundir dicho mejoramiento de tecnología en el regadío son las delegaciones de Sagarpa, también se difunde en el Diario Oficial de la Federación. Sin embargo, el medio propulsor de la participación en convocatorias son las instituciones federales, de ahí la importancia de conservar los vínculos políticos, a través de los representantes de los partidos políticos.

La Secretaría de Desarrollo Agropecuario (Sedagro) en el 2012 llevó a cabo el programa de Alta Productividad de Maíz. Bajo este programa se proyectó la modernización y tecnificación de algunas zonas de riego, como el Módulo de Riego Danxho del Distrito de Riego 44. El diagnóstico de eficiencia hídrica que la CNA reportaba para ese año, antes de aplicarse el programa, era que la distribución se realizaba por canales a cielo abierto, y la eficiencia del riego se estimaba en $35 \%$, por lo tanto, con el sistema tecnificado propuesto, ésta podría alcanzar $90 \%$ de eficiencia, con ello se rescatarían 16 millones de metros cúbicos, susceptibles de ser aprovechados en 2 mil 200 hectáreas adicionales a las 2 mil 800 que actualmente se regaban (Entrevista a Francisco Corona. Director de Infraestructura Rural/SEDAGRO, Febrero 2012).

El objetivo fue modernizar y tecnificar el sistema de riego, con lo cual más de 3 mil productores podrían disponer del vital líquido para regar más de 5 mil hectáreas, proyectada con una inversión tripartita de 500 millones de pesos. Se precisó que la obra consistiría en derivar de la presa Danxho el gasto necesario para la zona de riego a través de líneas entubadas con dispositivos de control y protección, dicha acción facilitará proporcionar el riego presurizado sin bombeo. La falta de correspondencia de datos entre burócratas de instituciones diferentes como la Sagarpa y la CNA se evidencia en la aseveración de que el proceso de modernización hidráulica ampliaría la frontera agrícola en poco más de 5,000 has cuando en realidad dicha superficie proyectada era la que contemplaba el módulo desde el siglo XX.

Cuando el Estado y las empresas que financian las obras asumen la toma de decisiones respecto a mejorar el riego mediante el entubamiento de agua se provoca una disrupción ambiental y social. En el primer caso se elimina la flora ribereña y aumenta para la población regante y no regante la escasez de agua doméstica —el agua del canal utilizada para suplir las deficiencias de agua doméstica, obtenida con un tiempo mínimo de acarreo, ahora tiene otros destinatarios y se encuentra fuera del alcance de los lugareños-; en el segundo, la asistencia técnica y mejora de producción agrícola se focaliza a productores grandes y medianos generando competencia por el recurso hídrico con los pequeños productores, los pequeños productores abandonan los terrenos de cultivo históricos por la limitación hídrica.

En el caso de la Unidades para el Desarrollo Rural de Chihuahua, el gobierno federal buscó promover, coordinar, vigilar, supervisar y formular todas las acciones relativas a programa federales como el siguiente lema: "Construcción y Rehabilitación de Infraestructura para la Captación, Almacenamiento, Conducción y Aplicación de Agua para Riego", incrementando y rehabilitando la red de distribución de canales, capacitando a los productores respecto a las necesidades hídricas de las plantas, así como las bondades de los sistemas de riego tecnificados, "con estos recursos se adquirirán 3,370 toneladas de cemento y se ejecutarán obras de revestimiento de canales y/o entubado de agua para riego para una longitud de 40 $\mathrm{km}$., beneficiando una superficie de 800 has y 320 productores" (CNA, 2010), es decir, agricultores con promedio de 2.5 hectáreas. 
La promoción y aplicación de tecnología de riego, como canales de plástico subterráneos, pone énfasis en productores convencidos de aplicar la reconversión de cultivos, ello incrementa los ingresos financieros de la propia Asociación Civil y de la CNA al pagar un $50 \%$ más de cuota por los riegos puntuales requeridos en las hortalizas. El pago de cultivos tradicionales oscila entre 10 y 80 pesos, las hortalizas y demás cultivos comerciales requieren un pago de 100 a 450 pesos por ha.

Además este distrito, en la última década, ha implementado cultivos bajo invernadero, lo que ha llevado a que la organización de usuarios demande mayores volúmenes de agua, de hecho ha sido autorizado por la CNA la entrega de volúmenes por encima de lo que se tiene concesionado $\left(12.156 \mathrm{~mm}^{3}\right)$, llegando incluso hasta los $22.867 \mathrm{~mm}^{3}$, cifra que se encuentra muy por encima del volumen sustentable de $18.35 \mathrm{~mm}^{3}$ calculado para este DR: $18 \mathrm{~mm}^{3}$. Este aumento de agua ha favorecido a algunos productores que no se ajustan a las reglas establecidas de turnos de agua, desfavoreciendo a los pequeños productores que deben vigilar y entablar constantes riñas con los empleados de aquellos usuarios con propiedad privada.

La situación prevista por la CNA ha sido priorizar en atender la demanda de agua de los usuarios que se incorporan en programas federales para reconvertir sus cultivos mediante la tecnificación de sistemas de regadío. Dicho implemento tecnológico afecta los almacenamientos secundarios como los bordos, éstos han apoyado a los agricultores contra las inundaciones de sus terrenos de cultivo y de casas habitación, sirviendo como espacios de contención de crecidas del agua. Es de destacar que los suelos que prevalecen en este espacio de riego requieren de mayores volúmenes de agua a los establecidos con las láminas brutas promedio.

El caso de otro DR considerado pequeño es el de Chiconahutla. Para 1958, la relación de propietarios de predios con pozos de riego cedidos para el acueducto de Chiconautla fueron: 38 usuarios, 92 pozos y 2523 ha (AHA, AN, caja: 1287, expediente: 17288, Fojas: 11, foja 9). Al ubicarse en la zona conurbada del Distrito Federal, en estos años, fueron afectados sus usos de agua de riego, permutadas las aguas claras (explotación subterránea) por aguas negras provenientes del desagüe de la Ciudad de México.

Se transcribe un escrito donde los vecinos de Santa María Chiconautla, municipio de Ecatepec de Morelos, solicitan se les concedan aguas negras para regar aproximadamente 250 has A partir de entonces los pueblos de San Pedro Atzompa, San Francisco Cuautliquixca y Santa María Ozumbilla reciben el beneficio de las aguas negras y que realmente son los de la zona donde el departamento del Distrito Federal perforó pozos para la dotación de agua potable a la Cd de México (AHA, AN, caja: 1282, expediente: 17200, Fojas: 12, foja 6). El total de superficie sembrada para el 2005 era de 2,709 has con cultivos de maíz forrajero, alfalfa, maíz de grano y avena. Dicha superficie ha ido reduciendo, quedando en 870 has en el 2012, el motivo son los altos costos de energía eléctrica requerida para extraer y bombear agua así como la eminente contaminación (CNA, 2007, 2012).

En 1986, se giraron instrucciones para reconstruir en forma provisional la represa en el $\mathrm{km}$ 27 del gran canal de desagüe, con el fin de asegurar el riego por bombeo de la unidad del sistema Chiconautla. "Asimismo se deberá insistir ante la dirección general de proyectos del SARH para que elabore el proyecto de la nueva obra de toma para Chiconautla a fin de que 
el Departamento del DF realice su construcción como parte del compromiso adquirido con SARH” (AHA, Infraestructura Hidráulica, caja:740, expediente: 21285, Foja 1).

Para tener un claro entendimiento de la función de nuevos implementos tecnológicos del riego, en el caso de DR se implementan Programas de Capacitación en Cascada, en el que los cursos impartidos son directamente en las oficinas del DR o en otros estados de la República. Los instructores son los subjefes administrativos de los DR, empresas contratadas por el DR o bien por la Asociación Nacional de Unidades de Riego. Dicho conocimiento técnico es volátil, porque los asistentes a cursos tienen una duración en el cargo no mayor a tres años, aunque también existen casos de reelección por tres periodos, sobre todo del presidente de la Asociación Civil.

Sin embargo, la asistencia a dichos cursos no se cumple cabalmente; la selección de usuarios y la constante rotación de los mismos impide que la adquisición de conocimiento sea vertida en un equipo de personas que capaciten constantemente a las nuevas autoridades. Otro ejemplo sobre tecnología hidráulica se muestra en la acción de la institución federal de la Secretaría de Desarrollo Agropecuario, ésta en el 2011 aportó 200 toneladas de cemento para abastecimiento de canales de riego, dicha gestión fue realizada por la CNA, en el caso de implementos para invernaderos se han logrado instaurar 6. Es decir, la transferencia de tecnología beneficio a 6 usuarios de un total de 3,735 usuarios (Entrevista a presidente de DR 033, Módulo IV. Miguel Ángel Vargas Benítez).

El caso de un DDR es el que corresponde a Coatepec Harinas, se tienen datos de riego históricos de 1929, fecha en que se documenta que los usuarios de pueblo de Acuitlapilco utilizaban las aguas del río Jabalí y el Chiquihuitero desde tiempo inmemorial, a través de un canal de 45 kilómetros de longitud, ellos solicitaban su confirmación de derechos a los usuarios de Pilcaya en Guerrero ateniéndose a que ambos pueblos obraban de común acuerdo (AHA, AS, caja 1329, exp 18091, legajo 1, foja 160).

Los cultivos principales en 1929 eran maíz de grano, frijol, camote, chile, cacahuate, tomate, guacamote y caña. Años más tarde al ser integrados cuatro municipios en este DDR se promueven más de veinte productos comerciales como: maíz forrajero, aguacate, alfalfa verde, café, caña de azúcar, ciruela, chile verde, durazno, frambuesa, fresa, granada, guayaba, lima, limón, mamey, mango, manzana, níspero, papaya, pastos y praderas, piña, plátano, zapote, zarzamora, pepino, chícharo, camote, calabacita, ebo, haba, papa, jitomate y flores de cuatro variedades. Menos de la mitad del territorio de dicho DDR (40\%) se dedica a la agricultura. Del total de hectáreas de superficie de riego la mitad de ellas son consideradas de medio riego. Los cultivos bajo riego tradicionales como el frijol y el cacahuate han asumido estrés hídrico. El frijol, por requerir un periodo de riego de tres meses por un periodo de ocho días, debido a que los terrenos son delgados de constitución areno-arcillosa; el caso del cacahuate, el periodo de riego dura seis meses y dan riegos cada quince días. En los últimos años dichas productores tradicionales compiten con los productores que apoya el gobierno federal.

Los sistemas de riego tradicionales se ubican en localidades Chiltepec, Zacanguillo, Ixtlahuaca de Villada, Primera del Monte, Acuitlapilco, San Luis y Cochisquila. Éstas están consideradas como localidades rurales, con menos de 1,000 habitantes. Es uno de los DDR que pese a la importancia de los cultivos presenta una alta desigualdad en la distribución de ingresos (Coneval, 2012). 
En el caso del módulo de riego de Temascalcingo, el cual riega con aguas negras, se presentan almacenamientos de tipo prehispánico como los jagüeyes, éstos suplen las deficiencias de aguas blancas. Sin embargo, es en dicho espacio donde la renta de la tierra se presenta con mayor fuerza, dejando las tierras que riegan con agua del río Lerma (altos niveles de concentración de contaminantes) en manos de los usuarios minifundistas. Los usuarios con dotaciones por accesión son los que rentan la tierra, incluida el agua, a personas que viven fuera de Temascalcingo para el cultivo de frutas como la fresa y otros cultivos como las flores. La búsqueda de terrenos con riego y topografía plana es la que permite en el DR visualizar un mercado de tierra y agua, a dicha presión por estos recursos se anexa la disminución de suelos agrícolas por la eminente contaminación del agua, estos factores limitan el aseguramiento del alimento.

Por lo tanto, los proyectos de transferencia de tecnología en sistemas de riego tradicional no se centran en la comunidad o en el sistema de riego (aquel espacio que incluye la fuente de agua, la red de canales y el espacio de riego), se abocan a algunos agricultores, aquellos que cumplen con ciertos parámetros financieros y jurídicos.

\section{Conclusiones}

El tema del agua es uno de los recursos esenciales de emancipación y participación de las organizaciones autogestivas en regadíos tradicionales frente al poder del Estado, los derechos de agua, sin embargo, al no ser derechos de propiedad de las comunidades, permiten que otro tipo de derechos particulares ejerzan presión sobre el recurso; de manera que las instituciones que intervienen en la asignación del agua no participan en las decisiones derivadas de los esquemas de distribución del agua o acompañamiento en el tema de acumulación excesiva de tierra que desfavorece a la clase ejidataria frente a la propiedad privada, por lo tanto, no es sorpresivo que la promoción de transferencia de tecnología esté focalizada a los usuarios con pretensiones y capital financiero para expandir no sus propiedades sino su producción, un nuevo modelo de acaparamiento de recursos esenciales como la tierra y el agua. La falta de reconocimiento de acción colectiva ante un bien de aprovechamiento común tiende a acrecentar la desigualdad social de los usuarios y su competencia por el recurso. A nivel de organizaciones autogestivas con regadíos tradicionales existe un derecho a la libertad de informarse y ser informados en torno a las decisiones. Por otra parte, las entidades que financian los proyectos de transferencia de tecnología desconocen derechos colectivos a la auto organización, lo que provoca una carencia de proyectos de conservación de tierras y aguas en los ejidos y comunidades con regadío tradicionales y población originaria.

La renta de tierras en ejidos que tienen dotación por accesión no sólo impacta en el sistema de tiempos de agua, además desliga a los nuevos productores de las actividades propias del sistema de riego tradicionales como la rehabilitación, conservación y su integración a los acuerdos colectivos.

\section{Referencias}

AHA (Archivo Histórico del Agua): Fondo: Infraestructura hidráulica, caja: 740, expediente: 21285 , foja 1. 
Burns, E. (coord.) (2009): Repensar la cuenca: la gestión de ciclos del agua en el Valle de México, México, 152 pp.

Castro, M., Karina Kloster y M.L. Torregrosa (2004): Ciudadanía y gobernabilidad en México: el caso de la conflictividad y la participación social en torno a la gestión del agua, México, pp. 339-369.

Cedillo Portugal, Eugenio y María Luisa Calzada Sandoval (2010): "Riego y semillas mejoradas en la agricultura moderna", Revista Encuentros, 8:1-19, FES Aragón, Universidad Nacional Autónoma de México.

Comisión Nacional del Agua (2013): Subdirección General de Infraestructura Hidroagrícola, Gerencia de Distritos de Riego. Padrón de beneficiarios del programa 079 "rehabilitación, modernización y equipamiento de distritos de riego", http://www.conagua.gob.mx/CONAGUA07/Noticias/Beneficiarios_2013_S079.pdf

Comisión Nacional del Agua (2012): Estadísticas agrícolas de unidades de riego año agrícola 2006-2007, México, Subdirección General de Infraestructura Hidroagrícola Gerencia de Unidades de Riego.

Comisión Nacional del agua (Conagua) (2008): Programa hídrico visión 2030 del organismo de cuenca valle de México y sistema Cutzamala, México, SEMARNAT, 197 pp.

Comisión Nacional del Agua (2007): Estadísticas agrícolas de los distritos de riego. Año agrícola 2005-2006, México, Comisión Nacional del Agua.

Consejo Nacional de Evaluación de la Política de Desarrollo Social (2012): Informe de pobreza y evaluación en el Estado de México, México, CONEVAL.

FAO (2000): El riego en América Latina y el Caribe. Informe sobre temas hídricos, División de Fomento de Tierra y Agua, Roma, ftp://ftp.fao.org/agl/aglw/docs/wr20.pdf. Consulto el 12 de enero de 2014.

Glick F. Thomas (2013): Irrigation and Society in Medieval Valencia, Harvard, Harvard University Press, pp. 408.

Glick F. Tomas (2007): Tecnología, ciencia y cultura en la España medieval / Islamic and Christian Spain in the Early Middle Ages, Alianza / Grupo Anaya Comercial, pp.167.

Glick F. Tomas (2005): Islamic and christian spain in the Early Middle Ages, Netherlands/Estados Unidos, Brill.

Gozzer Stefania (2010): Entrevista a Thomas Glick, Mètode. Revista de Difusión de la Investigación de la Universitat de València, 67, otoño.

Mata Olmo R. (2004): Agricultura, paisaje y ordenación del territorio, Polígonos, Revista de Geografia, 14:97-137.

Organización de las Naciones Unidas para la Agricultura y la Alimentación (FAO) (2002):

"Relaciones tierra-agua en cuencas hidrográficas rurales", Boletin de tierras y aguas, 9, Roma, FAO.

Palerm, Ángel (1972): Agricultura y sociedad en Mesoamérica, México, SEP.

Palerm, Angel y Juan Mestre (2007): Agna y agricultura: Angel Palerm, la discusión con Karl Wittfogel sobre el modo asiático de producción y la construcción de un modelo para el estudio de Mesoamérica, México, Universidad Iberoamericana.

Segura Bustamente, Mariano (1978): Aula-taller sobre transferencia de tecnología agrícola, Bib. Orton Instituto Americano de Ciencia Agrícolas (IICA / CATIE), Guatemala. 
Silva R. (2008): Hacia una valoración patrimonial de la agricultura, Scripta Nova. Revista Electrónica de Geografía y Ciencias Sociales, XII: 275, Barcelona, Universidad de Barcelona.

Torregrosa M.L, Kloster, K, Vera, J., Paré L. (2000): “Administración del agua”, en B. Jiménez, M.L.Torregros y A. Aboites (eds.), El agua en México: cances y encauces, Academia Mexicana de Ciencias / Conagua, pp. 595-624. 